\title{
Proton fluxes inside the South Atlantic Anomaly measured by the High-Energy Particle Detector (HEPD) on board the CSES-01 satellite during the 2018-2021 period
}

\section{Matteo Martucci ${ }^{a, *}$ on behalf of the LIMADOU-HEPD Collaboration}

(a complete list of authors can be found at the end of the proceedings)

\author{
${ }^{1}$ INFN - Sezione di Roma “Tor Vergata”, V. della Ricerca Scientifica 1, I-00133 Rome, Italy \\ ${ }^{2}$ University of Rome "Tor Vergata”, Department of Physics, V. della Ricerca Scientifica 1, I-00133 Rome, \\ Italy \\ E-mail: matteo.martucci@roma2.infn.it
}

Despite notable improvements made in the last decades, the characterization of the near-Earth proton radiation environment is still incomplete, with major uncertainties affecting the description of high-energy particles (>50 MeV) in the South Atlantic Anomaly (SAA) region. The HighEnergy Particle Detector (HEPD) on board the China Seismo-Electromagnetic Satellite (CSES01), launched on February 2018 on a Low-Earth Orbit and with an altitude of about $507 \mathrm{~km}$, is a light and compact payload suitable for measuring electrons (3-100 MeV), protons (30-300 $\mathrm{MeV}$ ), and light nuclei (up to a few hundreds of $\mathrm{MeV}$ ) with a high energy resolution and a wide angular acceptance. Thanks to its good identification performance, it can carry out precise and comprehensive measurement of particle fluxes, including angular information. The observations of HEPD could be fundamental not only for space weather purposes, but because they could help set important constraints on trapping and interaction processes in the Earth's atmosphere and magnetosphere. Furthermore, they enable the testing and validation of current theoretical and empirical models of the inner radiation belt, like the NASA AP9. In this contribution, we report a preliminary analysis of $>30 \mathrm{MeV}$ protons detected inside the SAA region between 2018 and 2021 .

$37^{\text {th }}$ International Cosmic Ray Conference (ICRC 2021)

July 12th-23rd, 2021

Online - Berlin, Germany

\footnotetext{
*Presenter
} 


\section{Introduction}

The radiation belts, also known as Van Allen belts, are regions of the Earth's magnetosphere where energetic charged particles are subject to long-term magnetic trapping. The outer belt is mostly populated by electrons with hundreds of $\mathrm{keV}$ to $\mathrm{MeV}$ energies, while the inner belt mostly consists of an intense radiation of energetic protons (from $\mathrm{MeV}$ up to a few $\mathrm{GeV}$ ), electrons/positrons (up to $\sim 8 \mathrm{MeV}$ ), and a minor component of ions $[1,2]$. Proton populations with energies above a few tens of $\mathrm{MeV}$ originate from the b-decay of free neutrons produced in the interaction between galactic cosmic-rays and the Earth's atmosphere in a mechanism called Cosmic Ray Albedo Neutron Decay (CRAND) [3, 4]. Since the discovery of the Van Allen radiation belts, after the launch of the first Explorer satellites in 1958 and the Pioneer in 1959 [5, 6], the scientific community has been considerably involved in modeling this space radiation environment [7, 8], particularly inside the region where the inner belt comes the closest to the Earth's surface - approximately located over the South Atlantic Ocean - known as South Atlantic Anomaly (SAA). The NASA AE9/AP9 set of models for high-energy electrons and protons, respectively, is the most recent one [9]. Despite their success in describing the radiation environment, both AE9 and AP9 are partly incomplete and often their predictions are not based on a statistically sufficient sample of direct measurements; thus, it is of key importance to test them and to provide new data-sets from in-flight instruments to improve their predictions.

\section{High-Energy Particle Detector and CSES Mission}

The China Seismo-Electromagnetic Satellite (CSES-01) [10] is the first of a series of multiinstrument monitoring satellites, scheduled for launch in the next few years. CSES-01 - put in orbit on February 2, 2018 - is currently flying on a sun-synchronous polar orbit at a $\sim 507 \mathrm{~km}$ altitude, with a 5-day revisiting periodicity. Among various scientific payloads, the High-Energy Particle Detector (HEPD), depicted in Fig.1, has been designed and built by the Limadou team, the Italian branch of the CSES Collaboration. It is a light and compact $(40.36 \mathrm{~cm} \times 53.00 \mathrm{~cm} \times 38.15$ $\mathrm{cm}$, total mass $\sim 45 \mathrm{~kg}$ ) payload, designed for electrons (3-100 MeV) and protons (35-250 MeV) measurements, and made up of a series of sub-detectors: a double-sided silicon microstrip tracking system, a plastic scintillator layer providing trigger and a range calorimeter - composed of a stack of 16 plastic scintillator in the upper portion (TOWER) and a $3 \times 3$ matrix of Lutetium-Yttrium Oxyorthosilicate (LYSO) inorganic scintillator crystals in the lower portion. The whole apparatus is surrounded by a total of 5 plastic scintillators (VETO) which reject particles not entirely contained in the detector. More technical details on the instrument can be found in [11, 12].

For protons inside the SAA - recognised by their characteristic signal deposited on the first scintillator plane $\left(\mathrm{P}_{1}\right)$ as a function of the total deposited energy (see Fig. 2 - the presence of trapped electrons is restricted to the $<8 \mathrm{MeV}$ energy range, thus not affecting the $35-250 \mathrm{MeV}$ energy range which has been analyzed in this work. Contamination from anti-protons is negligible [13] and no attempt was made to discriminate between other populations, like pseudo-trapped, quasi-trapped and precipitating protons or hydrogen isotopes, but it is worth noticing that they account for less than $10 \%$ of the proton population [14]. 


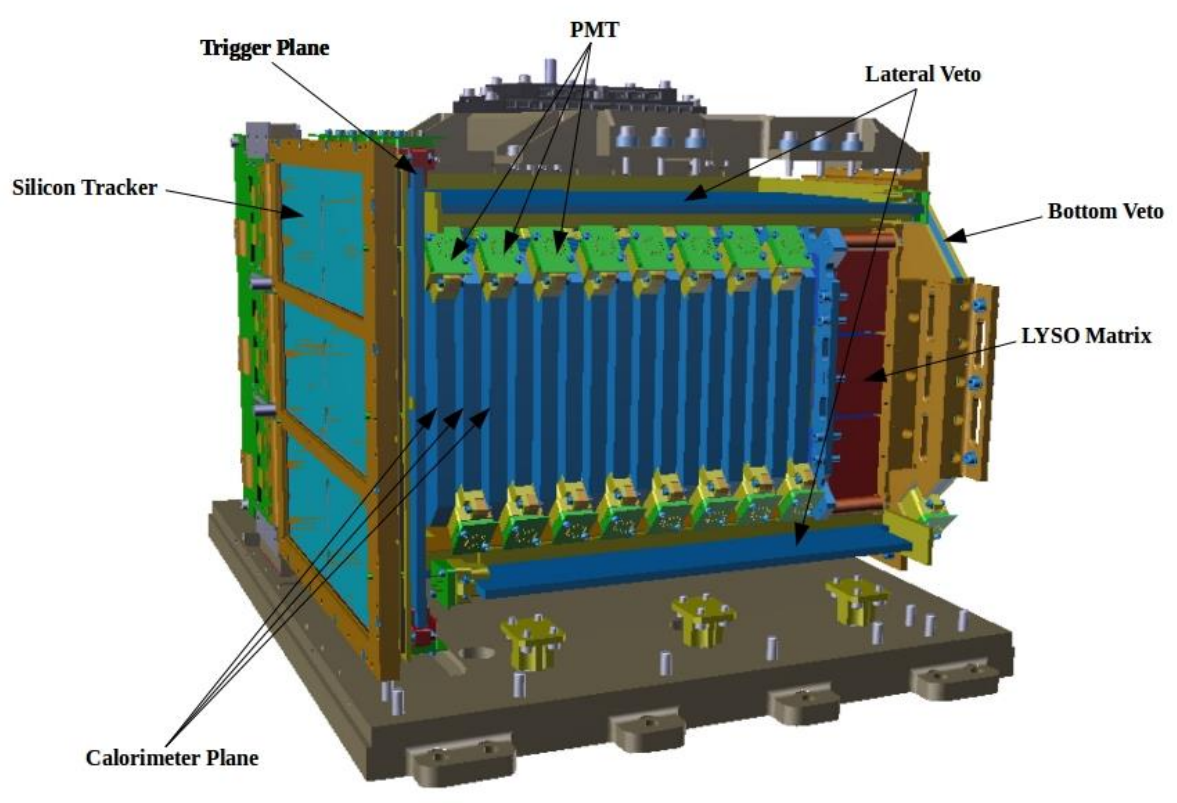

Figure 1: Schematic view of the HEPD detector.

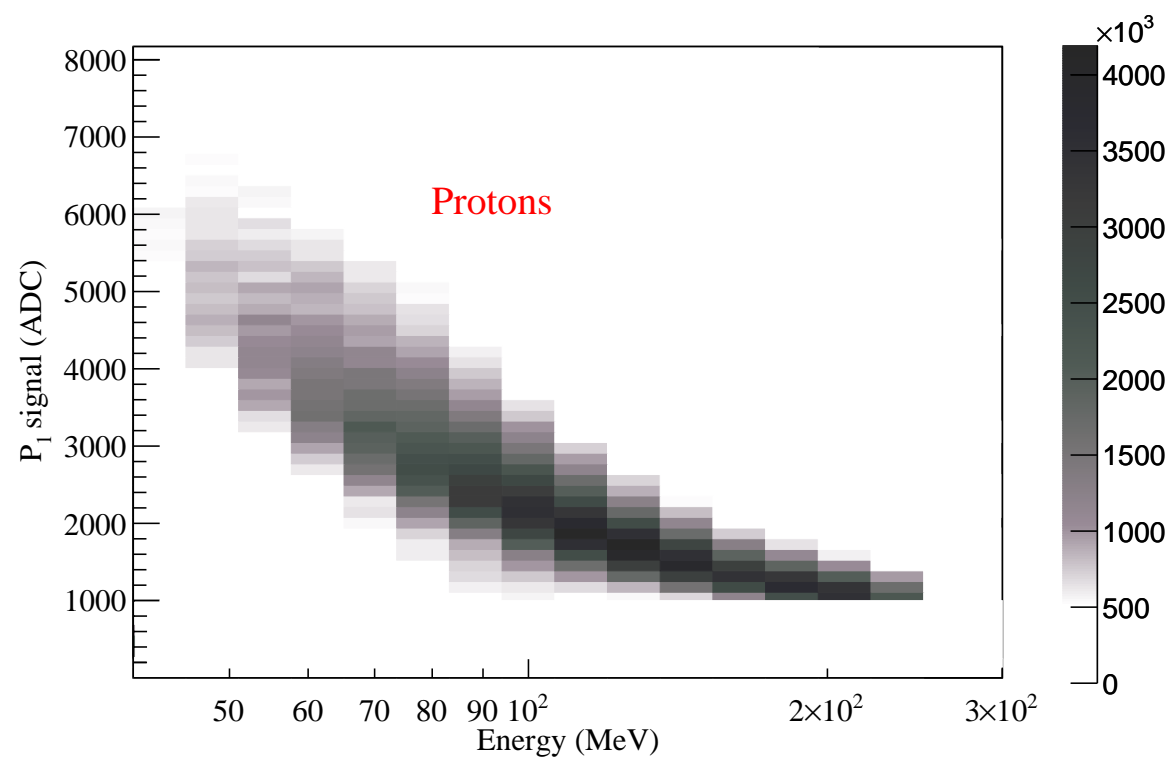

Figure 2: Protons ADC signal on the first plane of the calorimeter, $\mathrm{P}_{1}$, as a function of the total energy released inside the entire calorimeter itself. To better visualize the distribution of protons in the plot, only particles with vertical trajectories $\left(\theta<15^{\circ}\right)$ have been selected. 
$40 \mathrm{MeV}-45 \mathrm{MeV}$

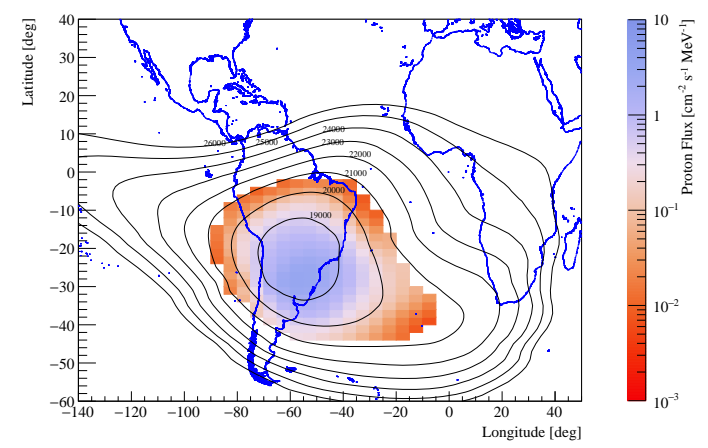

$200 \mathrm{MeV}-230 \mathrm{MeV}$

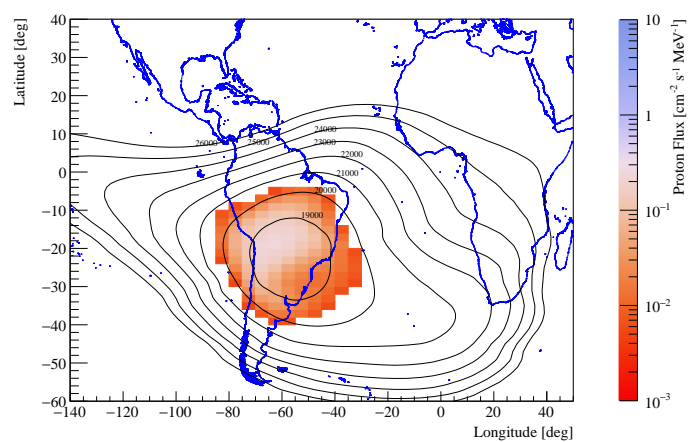

Figure 3: Geographical maps of omnidirectional proton fluxes (averaged over all local pitch angle) as a function of latitude and longitude for a low energy bin (40-45 MeV, left panel) and for a higher one (200-230 $\mathrm{MeV}$, right panel). In both panels, the isolines of the reconstructed magnetic field are also shown for clarity.

From a geographical/geomagnetic point of view, a selection on both the magnetic field $\mathrm{B}<20500$ (reconstructed using the IGRF-13 routines) and McIlwain parameter L-shell $<1.3$ (calculated using IRBEM libraries) is performed to operatively define the SAA region in which to collect the proton sample and compare data with AP9 models. It is crcial to understand that, due to the extreme anisotropy of trapped protons inside the anomaly, flux estimation is properly performed by using a multi-dimensional matrices approach. Therefore, final directional differential fluxes $F(\chi, E, \alpha)$ are obtained as:

$$
F(\chi, E, \alpha)=\frac{N(\chi, E, \alpha)}{2 \pi H(\Psi, E, \alpha) \Delta E \Delta \alpha \Delta \tau(\Psi)},
$$

where $N(\chi, E, \alpha)$ is the number of protons (corrected for selection efficiency) as a function of the satellite position $\chi=$ (longitude, latitude), reconstructed energy $E$ and local pitch angle $\alpha$, $\Delta \tau(\Psi)$ is the live time spent by CSES-01 at each orientation $\Psi$ and, finally, $H(\Psi, E, \alpha)$ is the effective area of the HEPD instrument. A resolution of $5^{\circ}$ for longitude, $3^{\circ}$ for latitude, $30^{\circ}$ for both $\theta$ and $\phi$ and $5^{\circ}$ for the local pitch angle, is employed.

Following this approach, for example, geographical maps of omnidirectional proton fluxes, as a function of latitude and longitude, are obtained, in 14 energy bins; their extension is depicted in Fig.3 for a low energy bin (40-45 MeV, left panel) and for a higher one (200-230 MeV, right panel).

More detailed will be given during the Conference, and they can be found in the poster associated to this proceeding.

\section{References}

[1] J.R. Cummings, A.C. Cummings, R.A. Mewaldt, R.S. Selesnick, E.C. Stone and T.T. von Rosenvinge, New Evidence for Anomalous Cosmic Rays Trapped in the Magnetosphere, in 23rd International Cosmic Ray Conference (ICRC23), Volume 3, vol. 3 of International Cosmic Ray Conference, p. 428, 1, 1993. 
[2] O. Adriani, G.C. Barbarino, G.A. Bazilevskaya, R. Bellotti, M. Boezio, E.A. Bogomolov et al., TRAPPED PROTON FLUXES AT LOW EARTH ORBITS MEASURED BY THE PAMELA EXPERIMENT, The Astrophysical Journal 799 (2015) L4.

[3] S.F. Singer, Trapped Albedo Theory of the Radiation Belt, Physical review Letters 1 (1958) 181.

[4] T.A. Farley and M. Walt, Source and loss processes of protons of the inner radiation belt, Journal of Geophysical Research 76 (1971) 8223.

[5] J.A. van Allen and L.A. Frank, Radiation Measurements to 658,300 Km. with Pioneer IV, Nature 184 (1959) 219.

[6] J.A. van Allen, C.E. McIlwain and G.H. Ludwig, Radiation Observations with Satellite 1958 $\varepsilon, J G R 64$ (1959) 271.

[7] S. Deme, G. Reitz, I. Apáthy, I. Héjja, E. Láng and I. Fehér, Doses Due to the South Atlantic Anomoly During the Euromir'95 Mission Measured by an On-Board TLD System, Radiation Protection Dosimetry 85 (1999) 301 [https://academic.oup.com/rpd/article-pdf/85/1-4/301/9924458/301.pdf].

[8] M. Martucci, R. Sparvoli, S. Bartocci, R. Battiston, W.J. Burger, D. Campana et al., Trapped proton fluxes estimation inside the south atlantic anomaly using the nasa ae9/ap9/spm radiation models along the china seismo-electromagnetic satellite orbit, Applied Sciences 11 (2021) .

[9] G.P. Ginet, T.P. O'Brien, S.L. Huston, W.R. Johnston, T.B. Guild, R. Friedel et al., AE9, AP9 and SPM: New Models for Specifying the Trapped Energetic Particle and Space Plasma Environment, Space Science Review 179 (2013) 579.

[10] X. Shen, X. Zhang, S. Yuan, L. Wang, J. Cao, J. Huang et al., The state-of-the-art of the China Seismo-Electromagnetic Satellite mission, Science in China E: Technological Sciences 61 (2018) 634.

[11] P. Picozza, R. Battiston, G. Ambrosi, S. Bartocci, L. Basara, W.J. Burger et al., Scientific Goals and In-orbit Performance of the High-energy Particle Detector on Board the CSES, Astrophysical Journal Supplements 243 (2019) 16.

[12] G. Ambrosi, S. Bartocci, L. Basara, R. Battiston, W.J. Burger, D. Campana et al., Beam test calibrations of the HEPD detector on board the China Seismo-Electromagnetic Satellite, Nuclear Instruments and Methods in Physics Research A 974 (2020) 164170.

[13] O. Adriani, G.C. Barbarino, G.A. Bazilevskaya, R. Bellotti, M. Boezio, E.A. Bogomolov et al., The Discovery of Geomagnetically Trapped Cosmic-ray Antiprotons, Astrophys. J. Lett. 737 (2011) L29.

[14] O. Adriani, G.C. Barbarino, G.A. Bazilevskaya, R. Bellotti, M. Boezio, E.A. Bogomolov et al., Reentrant albedo proton fluxes measured by the pamela experiment, Journal of 
Geophysical Research: Space Physics 120 (2015) 3728

[https://agupubs . onlinelibrary . wiley . com/doi/pdf/10.1002/2015]A021019]. 


\section{Full Authors List: LIMADOU-HEPD Collaboration}

S. Bartocci ${ }^{1}$, R. Battiston ${ }^{2,3}$, F. Benotto ${ }^{4}$, S. Beolè ${ }^{4,5}$, W.J. Burger ${ }^{3,6}$, D. Campana ${ }^{7}$, G. Castellini ${ }^{8}$, P. Cipollone ${ }^{1}$, S. Coli ${ }^{4}$, L. Conti ${ }^{1,9}$, A. Contin ${ }^{10,11}$, M. Cristoforetti ${ }^{12}$, L. De Cilladi ${ }^{4,5}$, C. De Donato ${ }^{1}$, C. De Santis ${ }^{1}$, F.M. Follega ${ }^{2,3}$, G. Gebbia ${ }^{2,3}$, R. Iuppa ${ }^{2,3}$, M. Lolli $^{11}$, N. Marcelli ${ }^{1,13}$, M. Martucci ${ }^{1,13}$, G. Masciantonio ${ }^{1}$, M. Mergé ${ }^{1, \dagger}$, M. Mese ${ }^{7,14}$, C. Neubuser ${ }^{3}$, F. Nozzoli ${ }^{3}$, A. Oliva ${ }^{11}$, G. Osteria $^{7}$, L. Pacini ${ }^{15}$, F. Palma ${ }^{1, \dagger}$, F. Palmonari ${ }^{10,11}$, A. Parmentier ${ }^{1}$, F. Perfetto ${ }^{7}$, P. Picozza ${ }^{1,13}$, M. Piersanti ${ }^{16}$, M. Pozzato ${ }^{11}$, E.

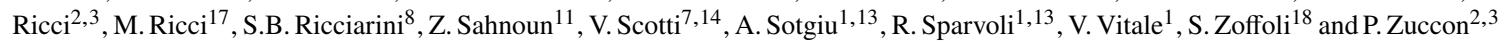

${ }^{1}$ INFN-Sezione di Roma "Tor Vergata”, V. della Ricerca Scientifica 1, I-00133 Rome, Italy;

${ }^{2}$ University of Trento, V. Sommarive 14, I-38123 Povo (Trento), Italy;

${ }^{3}$ INFN-TIFPA, V. Sommarive 14, I-38123 Povo (Trento), Italy;

${ }^{4}$ INFN-Sezione di Torino, Via P. Giuria 1, I-10125 Torino, Italy;

${ }^{5}$ University of Torino, Via P. Giuria 1, I-10125 Torino, Italy;

${ }^{6}$ Centro Fermi, V. Panisperna 89a, I-00184 Rome, Italy;

${ }^{7}$ INFN-Sezione di Napoli, V. Cintia, I-80126 Naples, Italy;

${ }^{8}$ IFAC-CNR, V. Madonna del Piano 10, I-50019 Sesto Fiorentino (Florence), Italy;

${ }^{9}$ Uninettuno University, C.so V. Emanuele II 39, I-00186 Rome, Italy;

${ }^{10}$ University of Bologna, V.le C. Berti Pichat 6/2, I-40127 Bologna, Italy;

${ }^{11}$ INFN-Sezione di Bologna, V.le C. Berti Pichat 6/2, I-40127 Bologna, Italy;

${ }^{12}$ Fondazione Bruno Kessler, V. Sommarive 18, I-38123 Povo (Trento), Italy;

${ }^{13}$ University of Rome "Tor Vergata", V. della Ricerca Scientifica 1, I-00133 Rome, Italy;

${ }^{14}$ University of Naples "Federico II", V. Cintia 21, I-80126 Naples, Italy;

${ }^{15}$ INFN-Sezione di Firenze, V. Sansone 1, I-50019 Sesto Fiorentino (Florence), Italy;

${ }^{16}$ INAF-IAPS, V. Fosso del Cavaliere 100, I-00133 Rome, Italy;

${ }^{17}$ INFN-LNF, V. E. Fermi 54, I-00044 Frascati (Rome), Italy;

${ }^{18}$ Italian Space Agency, V. del Politecnico, I-00133 Rome, Italy;

$\dagger$ At ASI Space Science Data Center (SSDC) also, V. del Politecnico, I-00133 Rome, Italy. 